\title{
Testes em condições para o controle de Dysmicoccus texensis (Tinsley) (Hemiptera, Pseudococcidae) em cafeeiro com nematóides entomopatogênicos do gênero Heterorhabditis (Rhabditida, Heterorhabditidae)
}

\author{
Viviane S. Alves ${ }^{1}$, Alcides Moino Junior ${ }^{2}$, Lenira V. C. Santa-Cecilia ${ }^{3}$, Cristiane Rohde ${ }^{2}$ \\ \& Marco Aurélio Tramontin da Silva ${ }^{2}$
}

1Departamento de Agronomia/Entomologia. Universidade Estadual de Londrina. Rua Vianna de Carvalho, nº 65. Bairro Vivendas do Arvoredo,
86047-520 Londrina-PR. vivialves21@ @otmail.com
²Departamento de Entomologia. Universidade Federal de Lavras. Caixa Postal 3037, 37200-000 Lavras-MG, Brasil. alcmoino@ufla.br;
crisrohde@ yahoo.com.br; marcotramont@ @otmail.com
${ }^{3}$ Epamig-CTSM/EcoCentro, 37200-000 Lavras-MG, Brasil. scecilia@epamig.ufla.br

\begin{abstract}
Tests for the control of coffee root mealybug Dysmicoccus texensis (Tinsley) (Hemiptera, Pseudococcidae) with Heterorhabditis (Rhabditida, Heterorhabditidae). Entomopathogenic nematodes (EPNs) have potential for biological pest control and have been successfully used in several countries in soil and cryptic pests control, as for example the coffee root mealybug Dysmicoccus texensis (Tinsley). Laboratory tests demonstrated that these agents are highly virulent to the insect, but semi-field and field tests are needed to determine their efficiency. Greenhouse tests were made in infested pots with two isolates and two application methods - dead insect bodies and aqueous suspension - in a complete randomized design with five replicates. Field tests were made in randomized plots (six plots) to evaluate six isolates of Heterorhabditis on coffee root for mealybug control. Greenhouse results demonstrate that aqueous suspension was more efficient for the two isolates, with $70 \%$ control efficiency for JPM3. In field experiments, treatments with aqueous suspensions of insecticide Actara 250 WG (thiamethoxam), used for comparison, and JPM3 were the only ones statistically different from control, with 81 and $65 \%$ control efficiency, respectively.
\end{abstract}

KEYWORDS. Biological control; coffee pests; greenhouse and field tests; microbial control.

RESUMO. Testes em condições para o controle de Dysmicoccus texensis (Tinsley) (Hemiptera, Pseudococcidae) em cafeeiro com nematóides entomopatogênicos do gênero Heterorhabditis (Rhabditida, Heterorhabditidae). Os nematóides entomopatogênicos (NEPs) apresentam potencial para o controle biológico de pragas e têm sido usados com sucesso, em vários países, no controle de pragas de solo e de ambientes crípticos, como a cochonilha-da-raiz-do-cafeeiro Dysmicoccus texensis (Tinsley). Testes de laboratório demonstram que estes agentes apresentam alta virulência sobre este inseto, no entanto, são necessários testes que avaliem a eficiência dos NEPs em condições de casa-de-vegetação e campo, sendo este o objetivo do presente trabalho. O experimento em condição de casa-de-vegetação para o controle da cochonilha foi realizado em vasos infestados, usando dois isolados do nematóide e dois métodos de aplicação (cadáver infectado e suspensão aquosa), conduzido em delineamento inteiramente casualisado com cinco repetições. O experimento em condições de campo foi conduzido em blocos casualisados (6 blocos), para avaliar a eficiência de dois isolados heterorhabditídeos no controle da cochonilha-da-raiz-do-cafeeiro. Os resultados mostraram que, em casa-de-vegetação, o método de suspensão aquosa apresentou melhores resultados para os dois isolados, sendo que JPM3 aplicado em suspensão aquosa foi o melhor tratamento, apresentando eficiência de controle de $70 \%$. No experimento de campo, apenas o tratamento com o inseticida Actara 250 WG (thiamethoxam), usado como padrão de comparação, e JPM3, aplicado em suspensão aquosa, diferiram da testemunha, apresentando 81 e $65 \%$ de eficiência de controle, respectivamente.

PALAVRAS-CHAVE. Controle biológico; pragas do cafeeiro; controle microbiano; testes em casa de vegetação e campo.

O uso de nematóides entomopatogênicos, como agentes de controle microbiano, ainda é limitado quando comparado com outros agentes, como fungos e bactérias (Grewal et al. 2001). No entanto, os nematóides apresentam uma combinação única de atributos que os tornam promissores no controle de vários insetos-praga (Grewal et al. 1999; Kaya \& Gaugler 1993; Shapiro-Ilan 2004; Georgis et al. 2006; Grewal et al. 2005).

Entre as principais vantagens apresentadas por estes organismos, está o fato de que apresentam a maior resistência a produtos fitossanitários que outros entomopatógenos, possibilitando sua utilização em programas de Manejo Integrado de Pragas (MIP); podem apresentar ação sinergística com outros agentes entomopatogênicos; apresentam boa capacidade de adaptação a novos ambientes, desde que estes não tenham condições adversas extremas; algumas espécies podem movimentar-se no ambiente, buscando pelo hospedeiro; podem reproduzir-se por partenogênese e são inócuos às plantas e outros animais, inclusive ao homem (Ferraz 1998; Shapiro-Ilan et al. 2005; Lewis et al. 2006).

Como limitações para o uso de nematóides 
entomopatogênicos no MIP, podemos citar a falta de produtos disponíveis no mercado. $\mathrm{O}$ desenvolvimento de um produto à base de um entomopatógeno passa por inúmeras fases, entre elas a caracterização e comprovação da eficiência do agente biológico, para o controle de determinado inseto-praga, e esses procedimentos requerem tempo e custos (Ferraz 1998). Além disso seu uso pode ser limitado também, em função do hábito de vida dos seus hospedeiros. Em geral, insetos que atacam partes aéreas das plantas e não tem contato com o solo em nenhuma fase do seu desenvolvimento, inviabilizam o contato com estes entomopatógenos, e consequentemente sua eficiência. Por outro lado, insetos com hábitos crípticos, ou insetos que passam pelo menos uma fase de sua vida no solo, potencializam a chance do encontro com os NEPs, e também a possibilidade de sucesso desses entomopatógenos no seu controle.

Nesse sentido, a cochonilha-da-raiz-do-cafeeiro, umas das principais pragas dessa cultura, tem demonstrado suscetibilidade aos nematóides entomopatogênicos, em função do seu hábito de vida críptico (vivem associadas às raízes da planta), em contato direto com o solo. Em trabalho realizado por Andaló et al. (2004) os resultados evidenciam a suscetibilidade deste inseto aos nematóides entomopatogênicos.

Estudos realizados em laboratório, como os sobre virulência e concentração letal são o princípio para a caracterização de isolados e sua utilização como agentes de um programa de controle (Alves et al. 1998; Pereira et al. 1998). Por outro lado, estudos em condições de casa-de-vegetação e campo também são importantes, pois retratam as possibilidades de sucesso de um nematóide, verificando sua eficiência no controle de um inseto-praga. Fatores como variação da temperatura, umidade, radiação solar, tipo de solo e busca pelo hospedeiro podem influenciar no sucesso de um agente de controle.

Assim, este trabalho teve como objetivos avaliar o desempenho de dois isolados heterorhabditídeos, previamente testados em condições de laboratório para o controle de Dysmioccus texensis (Tinsley) e duas formas de aplicação em condições de casa-de-vegetação e campo.

\section{MATERIALE MÉTODOS}

Criação de Dysmicoccus texensis. A criação de D. texensis foi conduzida no laboratório de Patologia de Insetos da Universidade Federal de Lavras (UFLA) em Lavras, MG e na Empresa de Pesquisa Agropecuária de Minas Gerais (EPAMIG), Centro Tecnológico do Sul de Minas, localizado no Campus da UFLA. A criação permaneceu em condições controladas de temperatura de $27 \pm 1^{\circ} \mathrm{C}$, com umidade relativa de $70 \pm 10 \%$ e fotofase de 12 horas, em câmara climatizada do tipo BOD. Como substrato para criação das cochonilhas foram utilizadas abóboras, (Cucurbita maxima), do tipo moranga, variedade Cabotcha, mantidas em bandejas plásticas. Para infestação de novas abóboras, foram colocados sobre elas, pedaços de uma abóbora já infestada, possibilitando a passagem das ninfas e adultos da cochonilha.
Nematóides entomopatogênicos. Os isolados CCA (Heterorhabditis sp.) e JPM3 (Heterorhabditis sp.) foram obtidos no Banco de Entomopatógenos do Laboratório de Patologia de Insetos da UFLA (Tabela 1), onde permaneceram armazenados em BOD em frascos Erlenmeyer na forma de suspensão aquosa, sob condições controladas a $16 \pm 1^{\circ} \mathrm{C}$, na concentração de até 500 juvenis infectantes (JI)/mL.

Quando necessário, a multiplicação dos nematóides foi feita em larvas de último instar da traça dos favos, Galleria mellonella (Linnaeus, 1758) (Lepidoptera, Pyralidae), provenientes do Laboratório de Patologia de Insetos do Departamento de Entomologia da UFLA.

Nesse processo de multiplicação foi utilizada a metodologia descrita por Poinar (1979), onde a infecção de larvas de último ínstar de G. mellonella com JIs é realizada por meio do sistema de infecção tópica. Após a aplicação, as larvas foram incubadas a $25 \pm 2{ }^{\circ} \mathrm{C}$ e após a morte foram transferidas para câmara seca. Armadilhas de White (1927) foram usadas para obtenção dos nematóides sob as mesmas condições. Para montagem dos experimentos os JIs emergidos foram coletados e armazenados sob temperatura de $16^{\circ} \mathrm{C}$ por no máximo cinco dias e em condições de aeração usando bombinhas de aquário.

Testes em casa-de-vegetação. Foram plantadas previamente 200 mudas de café, Coffea arabica L. cv. Mundo Novo, var. 476-4 com um ano de idade, em vasos com capacidade para 3 l, utilizando-se, como substrato solo, esterco bovino e adubo (NPK) na dosagem recomendada na região. As mudas foram produzidas na Fazenda Experimental de Lavras (FELA) da EPAMIG, em Lavras, MG.

A infestação das mudas com a cochonilha-da-raiz-docafeeiro foi feita, colocando um pedaço, de aproximadamente $4 \mathrm{~cm}^{2}$, de abóbora var. Cabotcha, infestada com adultos e ninfas da cochonilha $D$. texensis, junto ao colo da planta durante 3 dias. A verificação da infestação foi feita, escavando-se em volta do caule, na região do colo, e também pela presença de formigas doceiras, que são um indicativo da presença da praga nas plantas. Uma vez infestadas (após aproximadamente 60 dias), as mudas foram então submetidas aos tratamentos.

Isolados heterorhabditídeos previamente selecionados em condições de laboratório, foram aplicados através de duas formas: inoculação direta em suspensão aquosa no solo e pelo método de cadáver infectado, sendo aplicados na concentração de 28 e $29 \mathrm{JIs} / \mathrm{cm}^{2}$ (200 mL/vaso, aplicados próximo do colo da planta) e um cadáver infectado de $G$. mellonella/vaso (enterrada próxima do colo da planta) para os isolados CCA e JPM3, respectivamente.

A avaliação foi feita sete dias após a aplicação (durante esse período as plantas não foram regadas) através da contagem do número total de insetos vivos, em toda a área de raiz das plantas.

$\mathrm{O}$ experimento foi conduzido segundo o delineamento inteiramente casualizado, com cinco repetições, constituídas de uma planta cada. Os dados foram submetidos a teste de comparação de médias Scott-Knott $(\mathrm{P}<0,05)$. A eficiência de controle dos tratamentos foi calculada através da fórmula de Abbott (Alves et al. 1998). 
Testes em campo. Os testes, em condições de campo, foram conduzidos na propriedade Vista Alegre, no município de Garça, Estado de São Paulo ( $49^{\circ} 64^{\prime} \mathrm{O} / 22^{\circ} 23^{\prime} \mathrm{S}$ com altitude de $682 \mathrm{~m}$ ), sendo que a montagem do experimento foi no dia primeiro de junho de 2005 e a avaliação realizada no dia 30 do mesmo mês.

Foram utilizadas 36 plantas de café Coffea canephora Pierre \& Froehner, cv. Apoatã, de dois anos e meio de idade, infestadas naturalmente com $D$. texensis, sendo 6 destinadas para cada tratamento e cada planta foi considerada uma repetição. Os tratamentos consistiram da aplicação dos nematóides Heterorhabditis sp. JPM3 e Heterorhabditis sp.CCA, por duas formas de aplicação, a testemunha (onde foi aplicado água) e um inseticida padrão para comparação (Actara $250 \mathrm{WG}$ - thiamethoxam). Os blocos foram dispostos a partir da borda do cafezal, ao longo das linhas, sendo que cada bloco possuía seis plantas (uma repetição/tratamento). Para seleção das plantas infestadas, foi feita uma leve escavação próxima ao colo da planta, para verificar a presença ou não das cochonilhas. Como a infestação do campo não era homogênea, a distribuição das plantas dentro de cada bloco não foi contínua. Uma vez selecionadas as plantas infestadas, foi feito sorteio para a distribuição dos tratamentos dentro de cada bloco.

Todas as parcelas receberam $1 \mathrm{~L}$ de água antes de receberem os tratamentos. A aplicação do produto químico foi feita por inundação na concentração recomendada pelo fabricante $(0,23$ g p.c./planta em $80 \mathrm{~mL}$ de solução) (Souza \& Ribeiro 2003), sendo metade da calda aplicada de cada lado da planta. Os nematóides foram aplicados por dois métodos: inundação (da mesma forma que o produto químico) na concentração de $3,6 \times 10^{5} \mathrm{JIs} /$ planta $\left(100 \mathrm{JIs} / \mathrm{cm}^{2}\right)$ e pelo método de cadáver infectado (lagartas infectadas 8 dias antes da aplicação), sendo enterradas 10 larvas (cinco de cada lado da planta) a $10 \mathrm{~cm}$ de profundidade e a $5 \mathrm{~cm}$ do colo da planta. $\mathrm{O}$ tratamento testemunha recebeu apenas água.

Para transporte dos nematóides em suspensão aquosa, estes foram armazenados em erlenmayers de um litro de capacidade, mantidos em caixas de isopor refrigeradas e com aeração. Os cadáveres infectados foram transportados em placas de petri contendo papel filtro sob refrigeração.

A avaliação foi feita 30 dias após a aplicação, através da retirada de $2 \mathrm{~cm}^{2}$ de caule da região do colo, com auxílio de um estilete e feita a contagem do número de insetos vivos presentes. Devido o hábito criptico da cochonilha, o levantamento prévio do número de insetos presentes no campo se torna inviável, pois para tanto, seria necessária a remoção dos insetos. Assim, a comparação dos dados dos tratamentos foi feita apenas com os dados obtidos para a testemunha, que representa o número de insetos na ausência de tratamentos. Além disso, foi feita avaliação da persistência dos nematóides no campo, coletando-se $300 \mathrm{~g}$ de solo em volta das plantas, nas quais esses foram aplicados e também em torno das plantas do tratamento testemunha e produto químico. As amostras de solo foram encaminhadas ao laboratório de Patologia de Insetos do Departamento de Entomologia da UFLA em caixa térmica, onde foram submetidas à análise de persistência pela técnica isca-viva (Kaya \& Stock 2002), usando larvas de último ínstar de G. mellonella. Os dados foram submetidos à análise de variância e as médias comparadas pelo teste Scott-Knott $(\mathrm{P}<0,05)$. A eficiência de controle dos tratamentos foi calculada através da fórmula de Abbott (Alves et al. 1998).

\section{RESULTADOSEDISCUSSÃO}

Testes em casa-de-vegetação. No teste de patogenicidade de nematóides a $D$. texensis em vasos na casa-de-vegetação, não houve interação entre os fatores testados (isolados $\mathrm{x}$ método de aplicação).

O isolado JPM3, aplicado pelo método de suspensão aquosa alcançou valores significativos de controle de $68 \%$. Quando aplicado pelo método de cadáver infectado, a eficiência de controle diminuiu para apenas $46 \%$ (Tab. I). Por outro lado, o isolado CCA alcançou valor máximo de eficiência de $28 \%$, quando aplicado em suspensão aquosa, e de $18 \%$ quando aplicado pelo método de cadáver infectado (Tab. I).

Os resultados obtidos se devem, principalmente, à alta variabilidade existente entre diferentes espécies, ou mesmo entre diferentes isolados de nematóides entomopatogênicos, no que se refere ao comportamento e adaptações a condições ambientais. Estes dados, associados às características biológicas do inseto hospedeiro, são importantes para o estabelecimento de adequadas estratégias de controle (Georgis et al., 2006), salientando a importância da realização de testes em condições de casa-de-vegetação e campo.

Vários isolados avaliados como eficientes no controle de insetos em condições de laboratório, quando levados às condições de campo, podem não apresentar os mesmos resultados. Fatores do ambiente, do hospedeiro (comportamento séssil ou móvel, hábitos de vida, suscetibilidade), e do isolado, como capacidade de busca, especificidade ou não ao hospedeiro e resistência às condições ambientais desfavoráveis (Dowds \& Peters 2002) devem ser levados em consideração, na implementação de um programa de controle, tornando indispensável a realização de ensaios em campo.

Tabela I. Número médio de insetos vivos e porcentagem de eficiência de controle da cochonilha-da-raiz-do-cafeeiro em condições de casade-vegetação utilizando dois isolados (Heterorhabditis sp. CCA e Heterorhabditis sp. JPM3) através de dois métodos de aplicação.

\begin{tabular}{llclc}
\hline & \multicolumn{4}{c}{ Métodos de aplicação } \\
\cline { 2 - 5 } Isolados & \multicolumn{2}{c}{ Suspensão aquosa } & \multicolumn{2}{c}{ Cadáver infectado } \\
\cline { 2 - 5 } & $\mathrm{N}^{\circ}$ médio de & Eficiência & $\mathrm{N}^{\circ}$ médio de & Eficiência \\
& insetos/planta & $(\%)$ & insetos/planta & $(\%)$ \\
\hline CCA & $11 \pm 4,72 \mathrm{Aa}^{1}$ & 28 & $13 \pm 5,52 \mathrm{Aa}$ & 18 \\
JPM3 & $3 \pm 2,72 \mathrm{Ba}$ & 68 & $8 \pm 3,44 \mathrm{Aa}$ & 46 \\
Test. & $17 \pm 4,72 \mathrm{Aa}$ & - & $17 \pm 4,72 \mathrm{Aa}$ & - \\
\hline
\end{tabular}

${ }^{1}$ Médias seguidas de letras distintas maiúsculas nas colunas e minúsculas nas linhas diferem entre si pelo teste de Scott-Knott $(\mathrm{P}<0,05)$. 
Tabela II. Número médio de insetos vivos por planta e eficiência de controle de Dysmicoccus texensis em condições de campo, após aplicação do inseticida Actara e nematóides entomopatogênicos Heterorhabditis sp. CCA e Heterorhabditis sp. JPM3.

\begin{tabular}{ccc}
\hline Tratamentos & $\begin{array}{c}\mathrm{N}^{\circ} \text { médio de insetos/ } \\
2 \mathrm{~cm}^{2} \text { casca }\end{array}$ & $\begin{array}{c}\text { Eficiência } \\
(\%)\end{array}$ \\
\hline Actara WG 250 & $6 \pm 7,78 \mathrm{a}^{1}$ & 81 \\
JPM3 suspensão & $11 \pm 8,83 \mathrm{a}$ & 65 \\
JPM3 cadáver infectado & $24 \pm 19,50 \mathrm{~b}$ & 19 \\
CCA suspensão & $31 \pm 9,56 \mathrm{~b}$ & 0 \\
CCA cadáver infectado & $32 \pm 17,00 \mathrm{~b}$ & 0 \\
Testemunha & $30 \pm 10,00 \mathrm{~b}$ & - \\
\hline
\end{tabular}

${ }^{1}$ Médias seguidas de letras distintas nas colunas diferem entre si pelo teste de Scott-Knott $(\mathrm{P}<0,05)$.

Testes em campo. No experimento realizado a campo, os dados foram semelhantes aos encontrados no ensaio em casade-vegetação. Apenas o isolado JPM3, aplicado pelo método de suspensão aquosa, e o inseticida apresentaram valores de eficiência significativos no controle de D. texensis (65 e 81\%, respectivamente) (Tab. II). Quando aplicado pelo método de cadáver infectado, o isolado JPM3 apresentou 19\% de eficiência. O isolado CCA não foi eficiente em nenhum dos métodos de aplicação, não diferindo da testemunha.

Quanto à recuperação dos nematóides, com armadilha de isca viva, foi possível observar que, nos tratamentos que não receberam aplicação de nematóides (testemunha e inseticida), não houve nenhuma ocorrência destes agentes entomopatogênicos. Por outro lado, todos os tratamentos, em que houve aplicação de nematóides, apresentaram amostras positivas de isolamento (Tab. III).

Os melhores índices de recuperação, para os dois isolados, foram obtidos nos tratamentos de aplicação pelo método de suspensão aquosa (100\% para CCA e $83 \%$ para JPM3). Nos tratamentos de aplicação com cadáver infectado, o índice de recuperação foi menor, sendo 50\% para JPM3 e 17\% para CCA.

Vários fatores podem ter contribuído para a persistência dos isolados no campo, como por exemplo, a temperatura, umidade relativa do ar e do solo e radiação. De acordo com os dados do Instituto Nacional de Pesquisas Espaciais (INPE 2006), para o mês de junho de 2005, a precipitação máxima na cidade de Garça - SP foi de $80 \mathrm{~mm}$, com temperatura do ar

Tabela III. Recuperação de nematóides entomopatogênicos, Heterorhabditis sp. CCA e Heterorhabditis sp. JPM3, aplicados através de diferentes métodos para controle da cochonilha-da-raiz-do-cafeeiro Dysmicoccus texensis.

\begin{tabular}{lccc}
\hline Tratamentos & $\begin{array}{c}\text { Amostras } \\
\text { positivas }\end{array}$ & $\begin{array}{c}\text { Amostras } \\
\text { negativas }\end{array}$ & $\begin{array}{c}\text { Recuperação } \\
(\%)\end{array}$ \\
\hline Actara WG 250 & 0 & 6 & 0 \\
JPM3 suspensão & 5 & 1 & 83 \\
JPM3 Cadáver infectado & 3 & 3 & 50 \\
CCA suspensão & 6 & 0 & 100 \\
CCA Cadáver infectado & 1 & 5 & 17 \\
Testemunha & 0 & 6 & 0 \\
\hline
\end{tabular}

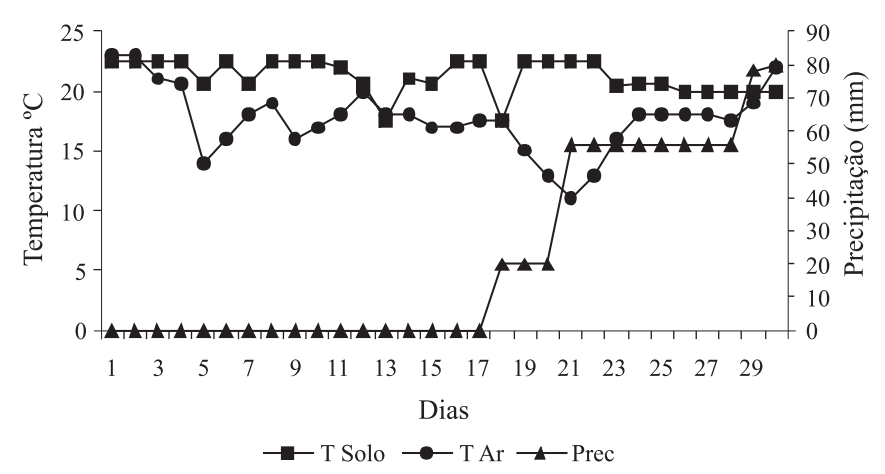

Fig. 1. Valores da precipitação pluviométrica acumulada, temperatura média do solo e temperatura média do ar para o mês de junho de 2005 na cidade de Garça - São Paulo (INPE 2006).

média de $20^{\circ} \mathrm{C}$ (Fig. 1). Esses fatores provavelmente contribuíram para a sobrevivência dos nematóides no campo, propiciando sua recuperação.

A eficiência dos nematóides entomopatogênicos, em condições de campo, requer umidade do solo adequada para sua sobrevivência. A ausência de umidade pode levá-los a dessecação, enquanto que o excesso de água no solo causa diminuição do oxigênio disponível e também dificuldade de movimentação (Kaya 1990; Koppenhofer et al. 1995; Peres et al. 2003). De acordo com os dados apresentados na Fig. 1, é possível observar que a precipitação acumulada no período em que o experimento foi conduzido, favoreceu os JIs aplicados.

A análise dos resultados deixa evidente a diferença entre os tratamentos. Entretanto, se ambos os isolados estavam presentes em ambas as formas de aplicação, então por que apenas JPM3 aplicado pelo método de suspensão aquosa foi eficiente no controle da cochonilha-da-raiz-do-cafeeiro? Uma possível explicação seria a forma de busca dos dois isolados, sugerindo que JPM3 provavelmente seja um isolado "cruiser", enquanto que CCA apresenta comportamento "ambusher". Segundo Lewis (2002), isolados do gênero Heterorhabtis podem apresentar ambas as formas de busca pelo hospedeiro, reforçando está hipótese.

Nematóides que possuem estratégia de busca do tipo "ambusher" são mais eficientes no controle de insetos que são ativos (que se movimentam mais), enquanto que nematóides que apresentam estratégia "cruiser" são mais eficientes no controle de insetos sésseis ou com hábitos crípticos (Lewis et al. 1992; Lewis 2002; Lewis et al. 2006).

Com relação aos métodos de aplicação avaliados, o uso de suspensão aquosa proporcionou melhores resultados quanto comparado ao método de cadáver infectado. Tais resultados diferem dos dados obtidos por Shapiro-Ilan et al. (2003), onde o autor conclui que o método de cadáver infectado é mais eficiente para o controle de Diaprepes abbreviatus (L.) e de Otiorhynchus sulcatus (F.). Segundo ele, o método de suspensão aquosa pode causar maior estresse físico nos nematóides durante o processo de armazenagem e durante o processo de aplicação. Além disso, o autor argumenta que o 
método de cadáver infectado favorece a manutenção da viabilidade e a capacidade de dispersão dos JI.

Da mesma forma, Del Valle (2008) avaliou a eficácia de Heterorhabditis baujardi aplicado pelo método de cadáveres infectados para o controle de Conotrachelus psidii em condições de casa-de-vegetação e campo, comprovando a suscetibilidade do inseto ao nematóide aplicado desta forma, em ambas as condições avaliadas. Por outro lado, o autor observou que formigas do gênero Ectatomma spp. podem realizar predação de até $80 \%$ dos cadáveres infectados usados para inoculação.

Dysmicoccus texensis possui associação mutualística com formigas do gênero Solenopsis, onde as mesmas são atraídas pela secreção do honeydew que é constantemente eliminado pela cochonilha, e em troca oferecem proteção e transportam as cochonilhas de uma planta a outra (Carter 1949; SantaCecilia et al. 2007). A ocorrência dessa associação sugere, que o mesmo detectado por Del Valle (2008), pode ter ocorrido, prejudicando o desempenho do nematóide aplicado pelo método de cadáver infectado.

Neste estudo, vários fatores indicam o isolado JPM3, como um agente promissor no controle de $D$. texensis, entretanto, testes mais detalhados ainda são necessários. Técnicas de aplicação em larga escala precisam ser avaliadas, testes sobre persistência dos JIs no campo, durante períodos maiores precisam ser feitos, bem como técnicas de produção e formulações adequadas que facilitem e disponibilizem tais recursos para o produtor. Mesmo assim, o isolado JPM3, aplicado em suspensão aquosa, teve uma eficiência similar ao do inseticida Actara, indicando-o como um agente promissor no controle da cochonilha-da-raiz-do-cafeeiro.

\section{REFERÊNCIAS}

Alves, S. B.; J. E. M. Almeida; A. Moino Jr. \& L. F. A. Alves. 1998. Técnicas de laboratório, p. 637-710. In: S. B. Alves. Controle Microbiano de Insetos. Piracicaba: FEALQ-USP, 1163 p.

Andaló, V.; A. Moino Jr.; L. V. C. Santa-Cecilia \& G. C. Souza. 2004. Seleção de isolados de fungos e nematóides entomopatogenicos para a cochonilha-da-raiz-do-cafeeiro Dysmicoccus texensis (Tinsley). Arquivos do Instituto Biológico 71: 181-187.

Carter, W. 1949. Insects notes from South America with special reference to Pseudococcus brevipes and mealybug wilt. Journal of Economic Entomology 42: 761-766.

Dowds, B. C. A. \& A. Peters. 2002. Virulence Mechanisms. p. 79-93. In: Gaugler, R. Entomopathogenic nematology. Wallingford: CABI. 388 p.

Del Valle, E. E. 2008. Utilização de cadáveres de Galleria mellonella (Lepidoptera: Pyralidae) infectados por Heterorhabditis baujardi LPP7 no controle do gorgulho-da-goiaba Conotrachelus psidii (Coleoptera: Curculionidae). Tese de doutorado apresentada a Universidade Estadual do Norte Fluminense Darcy Ribeiro-UENF. Campos do Goytacazes-RJ. 87 p.

Ferraz, L. C. C. B. 1998. Nematóides entomopatogênicos. p. 551567. In: S. B. Alves. Controle Microbiano de Insetos. Piracicaba, FEALQ-USP, 1163 p.
Georgis, R.; A. M. Koppenhofer; L. A. Lacey; G. Bélair; L. W. Duncan; P. S. Grewal; M. Samish; L. Tan \& R. W. H. M. Van Tol. 2006. Successes and failures in the use of parasitic nematodes for pest control. Biological Control 38: 103-123.

Grewal, P. S.; S. Converse; R. Georgis. 1999. Influence of production and bioassay methods on infectivity of two ambush foragers (Nematoda: Steinernematidae). Journal of Invertebrate Pathology 73: 40-44.

Grewal, P. G.; E. A. B. Nardo \& M. Aguillera. 2001. Entomopathogenic nematodes: Potential for exploration and use in South America. Neotropical Entomology 30: 191-205.

Grewal, P. S.; R. U. Ehlers \& D. J. Shapiro-Ilan. 2005. Nematodes as Biocontrol Agents. Wallingford: CABI, 528 p.

Instituto Nacional de Pesquisas Espaciais - INPE. 〈http://www.inpe.br>. Acesso em: 02 mar. 2006.

Kaya, H. K. 1990. Soil Ecology. p. 93-116. In: Gaugler, R.; H. K. Kaya. Entomopathogenic nematodes in biological control. Boca Raton: CRC Press, 350 p.

Kaya, H. K. \& R. Gaugler. 1993. Entomopathogenic nematodes. Annual Review of Entomology 38: 181-206.

Kaya, H. K. 2002. Natural enemies and other antagonists. p. 189-204. In: Gaugler R. (ed.). Entomopathogenic Nematology. CABI Publishing, Wallingford, UK. 388 p.

Koppenhofer, A. M.; H. K. Kaya \& S. P. Taormino. 1995. Infectivity of entomopathogenic nematodes (Rhabdita: Steinernematidade) at different soil depths and moistures. Journal of Invertebrate Pathology 65: 193-199.

Lewis, E. E.; R. Gaugler \& R. Rarrison. 1992. Entomopathogenic nematode host finding: response to host contact cues by cruise and ambush foragers. Parasithology 105: 309-319.

Lewis, E. E. 2002. Behavioral ecology. p. 205-224. In: R. Gaugler (ed.). Entomopathogenic nemathology. Wallingford: CABI, 388 p.

Lewis, E. E.; J. Campbell; C. Griffin; H. Kaya \& A. Peters. 2006. Behavioral ecology of entomopathogenic nematodes. Biological Control 38: 66-79.

Pereira, O. M.; S. B. Alves; D. R. Sosa-Gomes \& N. Macedo. 1998. Utilização de entomopatógenos no Manejo Integrado de Pragas. p. 1097-1115. In: S. B. Alves (Ed.). Controle Microbiano de Insetos. Piracicaba: FEALQ-USP. 1163 p.

Peres, E. E.; E. E. Lewis \& D. Shapiro-Ilan. 2003. Impact of the host cadaver on survival and infectivity of entomopathogenic nematodes (Rhabdita: Steinernematidae and Heterorhabditidae) under desiccating conditions. Journal of Invertebrate Pathology 82: 111-118.

Santa-Cecilia, L. C. V.; B. Souza; E. Prado; J. C. Souza \& M. J. Fornazier. 2007. Cochonilhas-farinhentas em cafeeiros: Reconhecimento e controle. Circular Técnica ${ }^{\circ}$ 8. Empresa de Pesquisa Agropecuária de Minas Gerais - EPAMIG.

Shapiro-Ilan, D. I.; E. E. Lewis; Y. Son \& W. L. Tedders. 2003. Superior efficacy observed in entomopathogenic nematodes applied in infected-host cadavers compared with application in aqueous suspension. Journal of Invertebrate Pathology 83: 270-272.

Shapiro-Ilan, D. I. 2004. Entomopathogenic nematodes and insect management. p. 781-784. In: J. L. Capinera (ed.). Encyclopedia of entomology. Dordrecht: Kluwer Academic Publishing, 580 p.

Shapiro-Ilan, D. I.; D. H. Gouge; S. J. Piggott \& J. P. Fife. 2005. Application technology and environmental considerations for use of entomopathogenic nematodes in biological control. Biological Control 38: 124-133.

Souza, J. C. \& J. A. Ribeiro. 2003. Cochonilha-da-raiz: cafeicultor, conheça e saiba como controlar esta praga com inseticidas neonicotinoides. Circular Técnica $n^{\circ} 162$. Empresa de Pesquisa Agropecuária de Minas Gerais - EPAMIG.

White, G. F. 1927. A method for obtaining infective nematode larvae from cultures. Science 66: 302-303. 